Philippe Haeck, né à Montréal le 27 décembre 1946. Tranquille jeunesse catholique jusqu'à vingt-cinq ans. Etudes universitaires en lettres. Mariage en 1968, deux enfants. Enseigne la littérature depuis mai 1968: donner la parole. Confondateur de la revue culturelle Chroniques en janvier 1975. Beaucoup de lectures de têtes chercheuses entre la tradition et la modernité: des rebelles, des femmes, des poètes, des philosophes, des moines, des psychanalystes. Des pò̀mes - un souffle court, une espèce de halètement qui rêve d'une respiration lente: Polyphonie. Roman d'apprentissage (1978), la Parole verte (1981), l'Atelier du matin (1987). Des textes critiques des engagements, des éclaircissements: l'Action restreinte. De la littérature (1975), Naissances. De l'écriture québécoise (1979), la Table d'écriture. Poéthique et modernité (1984). Publie chez VLB éditeur.

\title{
Blues clair
}

Elle chante une chanson folle. Je ne dis rien. Des notes rapides sur le piano, rapides. Affole j'entends une chanson ronde, haletant. Le piano, les notes, la voix éraflante. Quand je dis je suis affolé je le suis et ne le suis pas. Je mime l'affolement; quand la folie sera là je partirai à rire, son jeu me sera ouvert. Ainsi que sais-tu. Des pieds tassent ma vie. Qui me chante une berceuse. Fenêtre au sixième; tout le pont avec tous les gratte-ciel—carte postale jetable; on admire la carte. Fenêtre ouverte sur le ciel libre; on jette le ciel-trop large, comment le nommer, y danser. Des bouches rondes. Des lèvres retroussées. Des sons, des notes. Pas de sens, des cheveux propres, bien peignés. Signaux d'amérindienne. Cris clairs d'animaux imaginaires. Une note grave, intermittente. Es-tu. Tu aimes ta vie entre les plantes, tu te planques au milieu des voix-tu ne vas pas dans les café. Pas de spectacle. Rien que la voix large qui chante seule avec personne tout le monde une amie un ami. L'art de la conversation est perdu—on ne vend pas de billets.

Qui me caresse les pieds. Tant d'abeilles sur les fleurs. Sentir la terre s'exposer au soleil est plus utile que d'entendre la Symphonie pastorale.. La joie monte, tant de flèches tirées par l'archer en plein ciel. Tu marches sur un plateau avec Walter Benjamin, ton maître en solitude; «l'idée qui hante ce monde estudiantin allemand, tantôt plus tantôt moins, c'est qu'il faut profiter de sa jeunesse. [...] Ouvertement ou en secret, à la taverne ou dans le fracas des discours qui ponctuent les rassemblements, naît une ivresse chèrement payée et qu'on refuse de voir troublée.» Vous entrez dans les librairies d'occasion, rêvez au volcan éteint, le silence entre vous est bon comme les paroles, vous mangez un millefeuille au milieu d'un parc. Parce que vous savez que vous êtes vieux, votre âme se déplace comme le jeune arbre qui a tout à apprendre du ciel large, moutonneux: vous êtes prêts à vivre avec des enfants, à partager leurs jeux et leurs études-être tout ensemble créateur, philosophe, enseignant, amoureux. La terre bouillonne. On n'entend 
presque plus rien, toutes les forces de la nature paraissent se recueillir. L'archer sort son carquois, de grandes flèches bleues touchent le soleil vert. Le soleil gondole, les flèches le caressent de toutes parts, pas un coin de soleil qui n'éclate de joie. Une voix monte forte mêlée à une autre, lointaine et douce, tu ne reconnais pas les mots, tu penses les avoir déjà entendus; la première voix comme un hymne qui crève toute voûte, la seconde comme une chanson d'enfant inventée, petite voix rocailleuse pour dire c'est ma maman, c'est elle que j'aime, c'est elle qui m'ouvre les bras.

Ton ami est mort. Dans les café il s'assoyait à une table avec un cahier, des verres, un journal, un livre; ivre souvent au milieu du flot de paroles, fatigué de l'indifférence de presque tous et toutes, il parlait, écoutait avec un oeil qui disait ah la la la, invectivait, se confiait à un ami ou une inconnue. Dans la mort ce n'est plus qu'un petit enfant qui retourne d'où il est venu. Toi qui ne fréquentes pas les cafes - tu n'y aimes pas le bruit, tu n'y respires pas bien-tu l'aimais: l'amitié de celui qui allait dans les cafés et de celui qui n'y allait pas, de l'homme ivre et de l'homme discret: sobriété, ébriété se confondaient dans le goût commun du bonheur senti à travers des langages uniques. LES LIVRES SONT NOS AMIS. Ton ami est mort dans une ambulance-juste le temps de fumer un cigare avant de partir dans cette calèche qui le menait sur l'autre rive-, les musiques qu'il t'a données à entendre chantent en toi, la nécessité d'une parole critique et amoureuse est ton héritage. Un fantôme erre au Blues Clair, regarde les verres sur les tables, les corps, écoute les musiques, échange quelques mots avec qui est à côté de lui. Toi tu es ailleurs. Tu est resté au musée entre la bouche d'un homme et l'oreille d'une femme sur un pastel à l'huile rouge DO YOU KNOW HOW LONG IT TAKES FOR ANY ONE VOICE TO REACH ANOTHER. Tu rèves aux mots noirs, à la mer rouge qui sortent de la bouche des corps, au vers de Saint-Denys Garneau-«ll y a certainement quelqu' un qui se meurt»-, au porteur qui ressemble à celui qu'on a crucifié de s'être prétendu le fils du Père Amour, à ta vie qui ressemble à des cachiers d'esquisses et de notes, à cette phrase de Bertolt Brecht gravée sur une tablette de métal: OVERNIGHT, THE UNIVERSE HAS LOST ITS CENTRE AND NOW IN THE MORNING IT HAS ANY NUMBER OF CENTRES. NOW ANY POINT IN THE UNIVERSE MAY BE TAKEN AS A CENTRE. BECAUSE SUDDENLY THERE'S PLENTY OF ROOM. Son fantôme sourit, se lève, sort du Blues Clair, toi tu suis une petite musique noire au milieu de lettres de métal; lui s'en va rejoindre les prés des bisons infatigables, toi tu vas entendre l'idiot du village. Tu te sens proche de ce Don Quichotte dont la veste à queue et la queue de cheval sourient au milieu d'un rock-aquarium aux enfants qui lui demandent des histoires d'amour-à travers la solidité étrange des sons, l'illumination de la scène, la danse un peu folle des corps, le trésor apparaît. Quand tu sors dehors, marchent à côté de toi Meredith Monk, Patrick Straram le Bison ravi, Betty Goodwin, Michel Rivard; tu les ecoutes parler, fredonner, rire, tu aimerais les inviter à venir dormir chez toi. 